\title{
Defect detection with thermal imaging and phase shifting methods in lock-in thermography
}

\author{
Wontae $\mathrm{Kim}^{*}{ }^{*}$, Ranjit Shrestha ${ }^{*}$ and Manyong Choi ${ }^{* *}$ \\ *Department of Mechanical \& Automotive Engineering, Kongju National University, Cheonan, Republic of Korea, \\ sthranjit@gmail.com, ${ }^{\dagger}$ Corresponding Author: kwt@kongju.ac.kr \\ ${ }^{* *}$ Safety Measurement Center, Korea Research Institute of Standards and Science, Daejeon, Republic of Korea, \\ mychoi@kriss.re.kr
}

\begin{abstract}
This paper presents the results of experimental investigation of thermal phenomena in a square shape STS 304 specimen with artificial defects of varying depth and diameter at the back side. The material is aimed to be tested by means of lock-in thermography. The temperature field of the front surface of material tested is observed and analyzed at several excitation frequencies. Phase contrast method was used for better identification and analysis of the existing defects of the specimen. The phase contrast between the defective and sound regions illustrates the qualitative and quantitative investigation of defects. The two, three, four and five step phase shifting methods are investigated to obtain the information on defects. Phase contrast with four-step phase shifting at an optimum frequency of $0.01 \mathrm{~Hz}$ provides excellent results.
\end{abstract}

Keywords: Thermal imaging, lock-in thermography, STS 304, subsurface defects, phase shifting

\section{Introduction}

Steel is the material of choice for a large number and diverse industrial applications. High quality of material is an important aspect in several areas of human activities. The primary demand involves material characteristics and manufacturing processes, whereas the secondary demand requires periodic inspection with the foremost adequate nondestructive testing \& evaluation (NDT\&E) technologies.Among the emerging NDT\&E technologies, thermal imaging often known as infrared thermography (IRT) has gained much consideration in the scientific world because of outstanding progress in the production of high sensitive Infrared (IR) camera [1-4].

IRT is an emerging NTD\&E technique developed rapidly in recent years with many advantages such as high speed, large area of observation, wide area coverage and non-contact. IRT involves a remote mapping of temperature over test sample for finding out its surface and subsurface features with the emitted radiation from objects in the infrared band of the electromagnetic spectrum. To a greater extent,IRT have been used in the inspection of subsurface defects and features, thermo-physical properties, coating thickness and hidden corrosion, etc. The basic principle of active thermography consists of a thermal excitation of the sample to be inspected in order to produce a thermal contrast between the area of interest and the background. The local infrared emission from the surface is recorded by an infrared detector, and its analysis provides information about the structure of the investigated part [5-7]. It is widely known that IRT being an NDT\&E approach involves two classes, passive thermography and active thermography. In the coursework of the last few years, active thermography has been recognized as a powerful measurement tool for detecting surface and subsurface features in varieties of materials like metals, composites and polymers.

Depending on the heating procedure, active thermography could be further subdivided into various classes such as pulsed, step heating, lock-in, vibrothermography and pulsed phase thermography. Each technique has its own advantage and is chosen over the others depending on the type of defect or material to be analyzed The practice of using the lock-in thermography (LIT) technique widely improved the detection capacity of active IRT in the recent years. It involves, firstly, synchronizing the external excitation with IR camera acquisition, and secondly, in filtering parasitical infrared emissions. Therefore, experimental artifacts due to environmental reflections, local non-uniform emissivity of the sample surface, air turbulence, etc. are significantly reduced $[1,8,9]$

Phase-Shifting method is a technique used to determine the phase map from a set of thermographic images differing from each other by a known phase value. It is widely used in optical metrology because of its numerous advantages with features such as: speed and accuracy, point by point measurement, less sensitive to surface reflectivity variations and less sensitive to ambient light $[1,10,11]$.

In this study, the possibility of using LIT to quantitatively estimate the defects depth in STS 304 plate was explored. In order to determine the capability of LIT; an experimental setup was built in Thermal Design and Infrared Laboratory of Kongju National University, Cheonan, South Korea. The specimen developed by Korea Research Institute of Standard and Science (KRISS), South Korea has been used in the study. The experiment was carried out in a dark room and the room temperature was about $24^{\circ} \mathrm{C}$. Four cases of phase shifting methods were examined to extract the phase contrast of thermal wave harmonic components. 


\subsection{1/qirt.2016.057}

\section{Theory}

The periodical transfer of heat at the surface of a homogeneous semi-infinite material results in a thermal wave, which in one dimension is given by $[12,13]$.

$$
\mathrm{T}_{\mathrm{z}, \mathrm{t}}=\mathrm{T}_{0} \exp \left(-\frac{\mathrm{z}}{\mu}\right) \exp \mathrm{i}\left(\omega \mathrm{t}-\frac{\mathrm{z}}{\mu}\right)
$$

where, $\mathrm{T}_{0}\left[{ }^{\circ} \mathrm{C}\right]$ is initial change in temperature produced by the heat source, $\omega[\mathrm{rad} / \mathrm{s}]$ is the modulation frequency, $A(z)$ is the thermal amplitude, $\varnothing(\mathrm{z})$ is the phase, $\mathrm{f}[\mathrm{Hz}]$ is the frequency, $\lambda[\mathrm{m}]$ is thermal wavelength, $z$ [mm] is the defect depth, and $\mu[\mathrm{m}]$ is thermal diffusion length which determines the rate of decay of thermal wave as it penetrates through a material and defined by $[14,15]$.

$$
\mu=\sqrt{\frac{2 \alpha}{\omega}}=\sqrt{\frac{\alpha}{\pi f}}
$$

The phase angle calculation for two step phase-shfting method (2 SPSM), three step phase-shfting method (3 SPSM), four step phase-shfting method (4 SPSM) and five step phase-shfting method (5 SPSM) can be expressed by Eqs. (3), (4), (5) and (6) respectively $[1,10,11,16]$

$$
\emptyset(x, y)=\arctan \left(\frac{I_{2}}{I_{1}}\right)
$$

where $I_{1}$ and $I_{2}$ are thermpgraphic images shifted by $90^{\circ}$.

$$
\emptyset(x, y)=\arctan \left(\sqrt{3}\left(\frac{I_{3}-I_{2}}{2 I_{1}-I_{2}-I_{3}}\right)\right)
$$

where $I_{1}$ to $I_{3}$ are three thermographic images shifted by $120^{\circ}$ with reference phases of $0,2 \pi / 3,4 \pi / 3$.

$$
\emptyset(x, y)=\arctan \left(\frac{I_{2}-I_{4}}{I_{1}-I_{3}}\right)
$$

where $I_{1}$ to $I_{4}$ are 4 thermographic images with reference phase values of $0, \pi / 2, \pi$, and $3 \pi / 2$ corresponding to a phase shift of $90^{\circ}$.

$$
\emptyset(x, y)=\arctan \left(\frac{2\left(I_{2}-I_{4}\right)}{2 I_{3}-I_{5}-I_{1}}\right)
$$

where $I_{1}$ to $I_{5}$ are 5 thermographic images with reference phase values of $0, \pi / 2, \pi, 3 \pi / 2$ and $2 \pi$ corresponding to a phase shift of $90^{\circ}$.

Contrast to noise ratio (CNR) is used to distinguish the two regions of interest (ROI). Contrast is the absolute difference between the defective region information and the sound region information. Noise is the standard deviation of the sound area information. CNR analysis applied to each phase stepping method allows selection of most appropriate method. For this purpose, two region of interest (ROI)s, one for each defective region and one for adjacent to defective region (i.e. sound region), are selected. $\mathrm{ROI}$ in the defective region will be considered as "signal" $\left(\mathrm{D}_{\mathrm{ROI}}\right)$ and ROI in sound region will be considered as "noise" $\left(\mathrm{S}_{\mathrm{RO}}\right)$. The calculation of the CNR can be expressed as Eq. $(7)[17,18]$.

$$
C N R=20 \log _{10}\left(\frac{\left|D R O I_{\text {mean }}-S R O I_{\text {mean }}\right|}{\sigma}\right)
$$

where $\mathrm{DROI}_{\text {mean }}$ is the arithmetic mean of all the pixels inside the defective area; $\mathrm{SROI}_{\text {mean }}$ is the arithmetic mean of all the pixel inside the sound area and $\sigma$ is the standard deviation of the pixels inside the sound area. 


\subsection{1/qirt.2016.057}

\section{Methodology:}

Artificial defects (flat bottom holes) of different depths and diameters were created at the back side of the specimen. The schematic of the specimens with defect locations is shown in Fig. 1. For generation of sine waves of a single frequency, a programmable function generator (Agilent 33210A, Malaysia) was used and for detection of thermal waves infrared camera (SC645, FLIR Systems, Sweden) was used that has a $640 \times 480$ pixel resolution and sensitivity of $7.5-13 \mu \mathrm{m}$. The sample was heated with a sinusoidally modulated heat wave from two, $1 \mathrm{~kW}$ halogen lamps (connected to the output of function generator) kept at $100 \mathrm{~cm}$ away from the specimen. The camera was kept at a distance of $50 \mathrm{~cm}$ from the specimen in such a way that the axis of the camera coincides with the axis of the sample. Reflection method was adopted, i.e., images were acquired from the same surface that was heated periodically. A schematic of the general test arrangement is shown Fig. 2 [1$5,8]$.

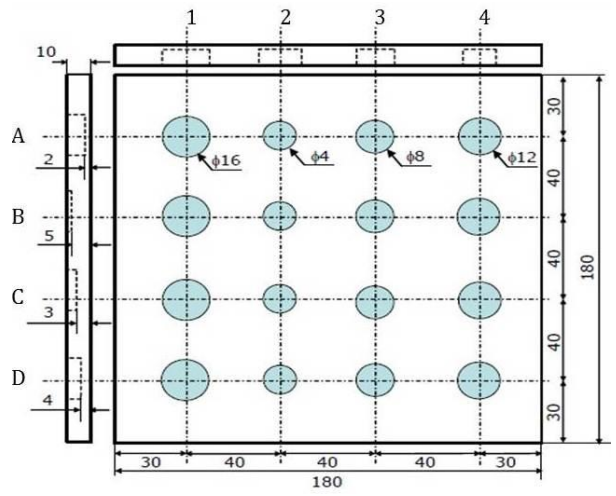

Fig.1. STS 304 Specimen with defects

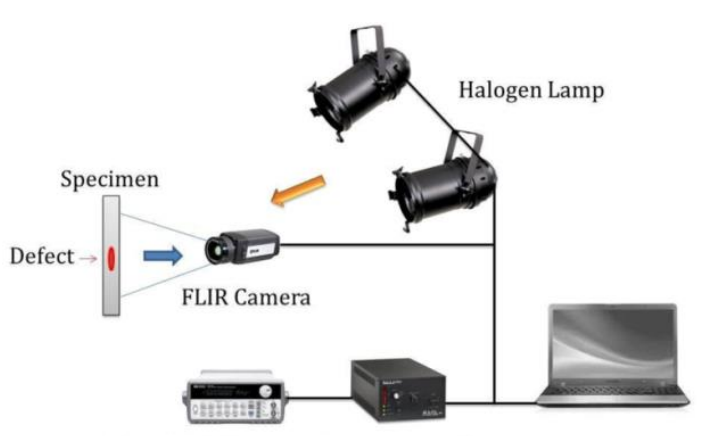

Function Generator Power Control Data Acquisition System

Fig.2. Schematic of experimental setup for LIT

\section{Results and Discussions}

During the experiments, the excitation frequency is chosen based on the diffusion length of the thermal signal and thermal images were captured by IR camera. The sequence of thermal images were monitored and recorded with respect to time. The experimental analysis was carried out for the 4 complete excitation cycles for each frequency ranging from 0.182 $\mathrm{Hz}$ down to $0.01 \mathrm{~Hz}$. The phase shifting methods were used in post processing every pixel of thermal images using the MATLAB programming language.

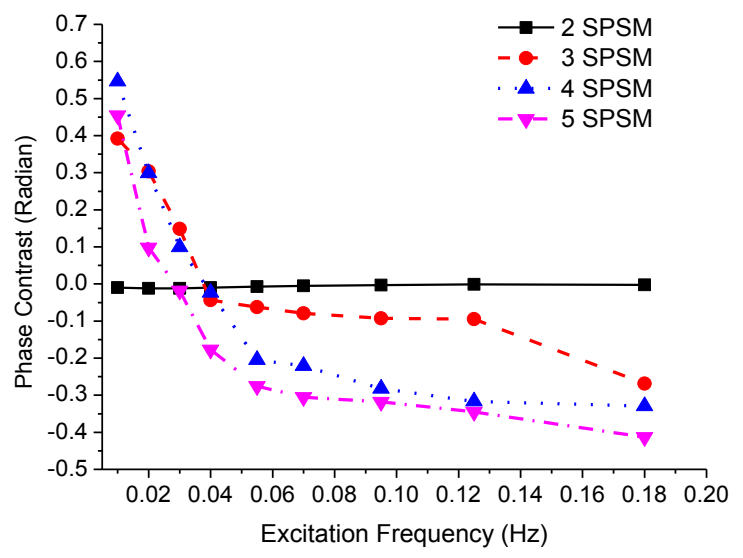

Fig.3. Phase contrast vs. excitation frequencies with various phase shifting methods

For the comparison of detection capability of each technique, phase contrast for the selected defect from the phase image at different excitation frequency was analyzed. Phase contrast was evaluated by subtracting the phase value located 


\subsection{1/qirt.2016.057}

centrally over the defects from the phase value measured in the sound area near the defects. A region of interest is selected and the phase value of each pixel within the region of interest is averaged to reduce the noise and error in measurement. The defect $A_{1}$ with diameter $16 \mathrm{~mm}$ and depth $2 \mathrm{~mm}$ is chosen for the analysis.

The Fig. 3 shows the phase contrast for the selected defect using different phase shifting methods with respect to excitation frequencies. From the result, it is found that two step phase stepping method is not suitable for the defect detection since there seems no phase contrast between the defected area and sound area. But in case of three, four and five step phase shifting methods, they follow the same trend line and it is observed that phase contrast increases when the excitation frequency decreased. So it is observed that at least 3 thermographic images should be used from the same modulation cycle to obtain the effective phase contras

CNR analysis applied to each phase shifting methods mentioned above enables to determine which method is more appropriate. The defect $A_{1}$ with diameter $16 \mathrm{~mm}$ and depth $2 \mathrm{~mm}$ is chosen for the analysis. The Fig. 4 shows the CNR of the selected defect with each phase shifting method with respect to excitation frequency. For the detection of defects CNR value should be higher than $0 \mathrm{~dB}$, so two point phase shifting technique was found inappropriate. From the analysis, it is found that four and five step phase shifting methods are preferable.

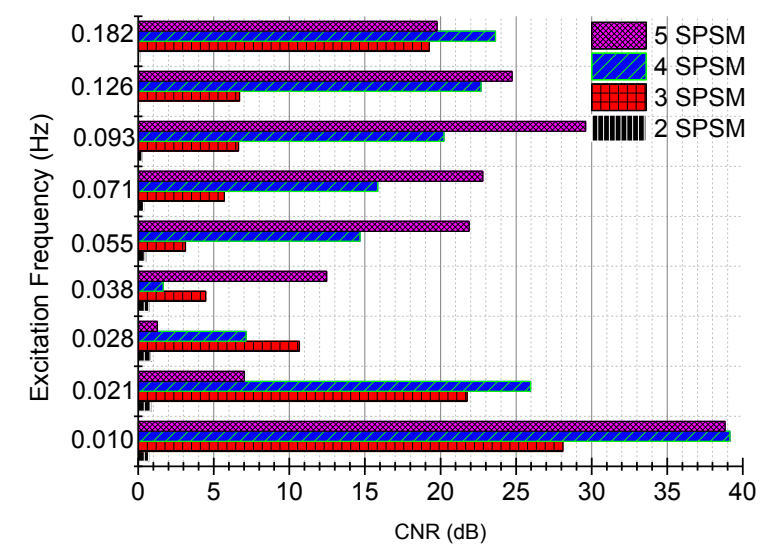

Fig.4. Phase contrast vs. excitation frequencies with various phase shifting methods

The effect of defect depth on phase contrast was analysed with 4 SPSM. Defects $A_{1}, C_{1}, D_{1}$ \& $B_{1}$ with depth $2,3,4$ $\& 5 \mathrm{~mm}$ respectively and the fixed diameter $16 \mathrm{~mm}$ were selected for the analysis on effect of defect depth on phase contrast. The phase contrast measured over the defects with respect to defect depth for a thermal excitation frequency of $0.01 \mathrm{~Hz}$ is shown in Fig.5. As can be seen in Fig.5, the phase contrast decreases with the increase in depth of defect for the fixed defect size. It can be observed that the curve follows a non linear trend and polynomial of order 3 gives best fit. The defect depth can be expressed as a function of phase, and the function is as expressed as, $13.34-63.59 \emptyset+140.63 \emptyset^{2}-113.97 \emptyset^{3}$.

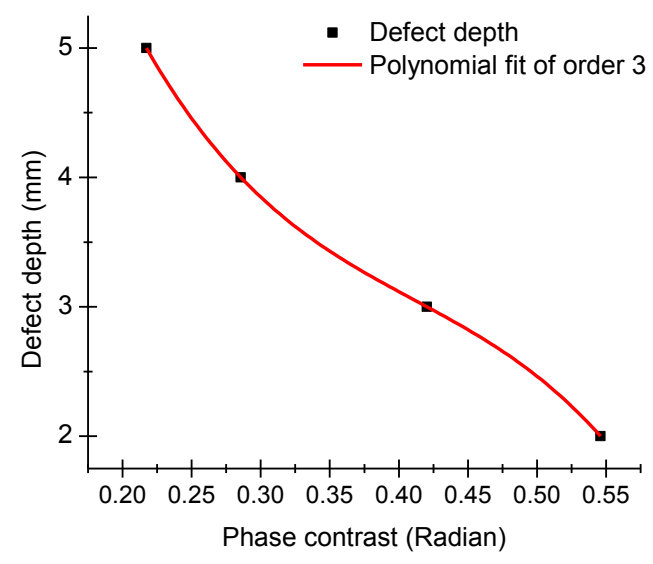

Fig.5. Defect depth calibration curve 


\subsection{1/qirt.2016.057}

\section{Conclusion}

This study explored the use of thermal wave imaging with LIT and various phase shifting algorithms. It is found that the correct excitation frequency range has to be selected for the material to detect the defects. The phase contrast method was used to determine the phase difference between the defective and sound area. The four step phase shifting method provides excellent results in the optimum frequency $0.01 \mathrm{~Hz}$. From the analysis of results, it may be inferred that LIT is able to visualize defects of different sizes and depth. The investigation shows that LIT could be a reliable non-destructive method for detecting the defects and can be employed as a preventive technique.

\section{Acknowledgement}

This work was supported by National Research Foundation of Korea (NRF-2014R1A1A2054595) funded by Ministry of Education, Science and Technology, South Korea and by the Human Resources Development program (No. 20154030200940) of the Korea Institute of Energy Technology Evaluation and Planning (KETEP) grant funded by the Ministry of Trade, Industry and Energy, Republic of Korea.

\section{REFERENCES}

[1] R. Shrestha, J. Park, and W. Kim, "Application of thermal wave imaging and phase shifting method for defect detection in Stainless steel," Infrared Physics \& Technology, vol. 76, pp. 676-683, 2016.

[2] S. Ranjit, K. Kang, and W. Kim, "Investigation of lock-in infrared thermography for evaluation of subsurface defects size and depth," International Journal of Precision Engineering and Manufacturing, vol. 16, no. 11, pp. 2255-2264, 2015.

[3] S. Ranjit and W. T. Kim, "Detection of subsurface defects in metal materials using infrared thermography; image processing and finite element modeling," Journal of the Korean Society for Nondestructive Testing, vol. 34, no. 2, pp. 128134, 2014.

[4] S. Ranjit and W. Kim, "Research on Defects Detection by Image Processing of Thermographic Images," International journal of Engineering and Technology, vol. 7, no. 5, pp. 1849-1855, 2015

[5] S. Ranjit, M. Choi, and W. Kim, "Quantification of defects depth in glass fiber reinforced plastic plate by infrared lock-in thermography," Journal of Mechanical Science and Technology, vol. 30, no. 3, pp. 1111-1118, 2016.

[6] S. Ranjit, W. Kim, and J. Park, "Numerical Simulation for Quantitative Characterization of Defects in Metal by using InfraRed Thermography," International Journal of Applied Engineering Research, vol. 9, no. 24, pp. 29939-29948, 2014.

[7] M. Streza, D. Dadarlat, Y. Fedala, and S. Longuemart, "Depth estimation of surface cracks on metallic components by means of lock-in thermography," Review of Scientific Instruments, vol. 84, no. 7, pp. 074902, 2013.

[8] S. Ranjit and W. Kim, "Detection and Quantification of Defects in Composite Material by Using Thermal Wave Method," Journal of the Korean Society for Nondestructive Testing, vol. 35, no. 6, pp. 398-406, 2015.

[9] G. Jinlong, L. Junyan, W. Fei, and W. Yang, "Inverse heat transfer approach for nondestructive estimation the size and depth of subsurface defects of CFRP composite using lock-in thermography," Infrared Physics \& Technology, vol. 71, pp. 439-447, 2015.

[10] S. Zhang, "Composite phase-shifting algorithm for absolute phase measurement," Optics and Lasers in Engineering, vol. 50 , no. 11, pp. 1538-1541, 2012.

[11] Y. Surrel, "Design of algorithms for phase measurements by the use of phase stepping," Applied Optics, vol. 35, no. 1, pp. 51-60, 1996.

[12] C. Meola and G. M. Carlomagno, "Recent advances in the use of infrared thermography," Measurement science and technology, vol. 15, no. 9, pp. R27, 2004. 


\subsection{1/qirt.2016.057}

[13] G. Busse, "Lockin-Thermography: Principles, NDE-applications, and trends," .

[14] H. Czichos, Handbook of Technical Diagnostics: Fundamentals and Application to Structures and Systems. Springer Science \& Business Media, 2013.

[15] X. Maldague, "Theory and practice of infrared technology for nondestructive testing," , 2001.

[16] K. Creath, "Phase-measurement interferometry techniques," Progress in optics, vol. 26, no. 26, pp. 349-393, 1988.

[17] R. Hidalgo-Gato, J. Andrés, J. M. López-Higuera, and F. Madruga, "Quantification by signal to noise ratio of active infrared thermography data processing techniques," Optics and Photonics Journal, vol. 3, no. 04, pp. $20,2013$.

[18] V. P. Vavilov and D. D. Burleigh, "Review of pulsed thermal NDT: physical principles, theory and data processing," NDT \& E International, vol. 73, pp. 28-52, 2015. 